\title{
Imaging in Sarcoma
}

\author{
Janet F. Eary ${ }^{1}$ and Ernest U. Conrad ${ }^{2}$ \\ ${ }^{1}$ Departments of Radiology and Orthopedics, University of Washington, Seattle, Washington; and ${ }^{2}$ Pediatric Orthopedics, Department \\ of Orthopedics and Sports Medicine, Seattle Children's Hospital, University of Washington, Seattle, Washington
}

\begin{abstract}
Learning Objectives: On successful completion of this activity, participants should be able to describe (1) the types of sarcomas and associate them with age groups for occurrence; (2) the uses of ${ }^{18} \mathrm{~F}-\mathrm{FDG}$ PET in sarcoma diagnosis and treatment response imaging; and (3) new areas of biologically specific PET imaging techniques that are likely to be relevant in sarcoma risk for poor outcome stratification.

Financial Disclosure: The authors of this article have indicated no relevant relationships that could be perceived as a real or apparent conflict of interest.

CME Credit: SNM is accredited by the Accreditation Council for Continuing Medical Education (ACCME) to sponsor continuing education for physicians. SNM designates each JNM continuing education article for a maximum of 1.0 AMA PRA Category 1 Credit. Physicians should claim only credit commensurate with the extent of their participation in the activity.

For CE credit, participants can access this activity through the SNM Web site (http://www.snm.org/ce_online) through December 2012.
\end{abstract}

Imaging has contributed to diagnosis and treatment decision making for patients with sarcomas, a highly heterogeneous tumor group. Derived from mesenchymal tissues, sarcomas occur in all parts of the body and in all patient age groups, with a highly variable histologic appearance and behavior. They are commonly separated into soft-tissue-derived and bone-derived groups; however, many exceptions to these classifications are seen clinically. Tumor size, cellular type and differentiation, and presence of necrosis are tumor features that can be used clinically to predict the risk of aggressive behavior. At present for soft-tissue sarcomas, the $5-y$ survival is approximately $60 \%$, with substantially better survival for patients with bone tumors, particularly pediatric patients. Imaging with nuclear medicine techniques plays an important role in diagnosis, treatment planning, and follow-up. Conventional 99mTc-methylene diphosphonate bone scans, ${ }^{201} \mathrm{TI}$ tumor imaging, and PET techniques have contributed to the care of sarcoma patients. Newer PET techniques with biologically specific imaging agents have also been evaluated to characterize sarcomas and contribute to risk stratification for poor outcome.

Key Words: sarcoma; PET; diagnosis; treatment response imaging

J Nucl Med 2011; 52:1903-1913

DOI: 10.2967/jnumed.111.092999

\section{$\mathbf{S}$}

arcoma is a cancer that originates from mesenchymal body tissues (1). These tissues comprise connective tissue, muscle, fat, bone, cartilage, peripheral nerves, and blood

\footnotetext{
Received Jul. 14, 2011; revision accepted Oct. 5, 2011.

For correspondence or reprints contact: Janet F. Eary, University of Washington Medical Center, Department of Radiology/Division of Nuclear Medicine, 1959 NE Pacific St., Room AA011, Box 356113, Seattle, WA 98195-6113.

E-mail: jeary@u.washington.edu

Published online Nov. 3, 2011

COPYRIGHT @ 2011 by the Society of Nuclear Medicine, Inc.
}

vessels. Sarcomas most commonly occur in muscles, bones, fat, and connective tissues. Although the bone marrow is also derived from mesenchymal tissues, hematologic malignancies are considered separately from solid tumors, or sarcomas. Sarcomas are a complex group of tumors that have many different clinical manifestations, but they most often present as a mass.

Sarcomas occur in both children and adults, and they constitute approximately $1 \%$ of cancers that occur among adults (2). In the United States, approximately 10,000 people are diagnosed with sarcomas each year. Additionally, approximately 3,700 people per year succumb to their disease (2). The median age of adult sarcoma patients is 59-60 y, and there are approximately 30 common sarcoma subtypes that occur in both adults and younger age groups (3). Most adult sarcomas are the soft-tissue type, with bone tumors and cartilage tumors occurring less frequently. Overall, there is a preponderance of women afflicted by sarcoma; women comprise approximately $60 \%$ of the patient population (3). Although the overall rate of cancer incidence is much lower for children, sarcomas constitute approximately a fifth of pediatric cancer diseases. Most childhood sarcomas are bone sarcomas, which differ from childhood soft-tissue sarcomas in clinical presentation, age preponderance, and outcome.

The complexity of clinical presentations and diagnoses for sarcomas is based on their pathologic characteristics. Because these tumors are derived from mesenchymal elements, at presentation the tumors may have histologic and tissue marker characteristics that identify the tissue of origin. The tumors can arise in any mesenchymal tissue, but for some, a cell of origin in normal tissues cannot be identified, supporting the theory that sarcomas form and may differentiate according to surrounding tissue signals or other environmental and genetic factors. There are more than 50 histologic subtypes of sarcoma (4). They most 
commonly occur in the extremities and trunk. Table 1 lists the most common soft-tissue sarcoma subtypes in adults, with the frequency of occurrence within the group for the more common types (5). Table 2 lists the most common pediatric sarcomas. Bone tumors predominate in the pediatric population.

Adults with sarcomas have an approximately 50\% 5-y overall survival rate (1). Patients with poor outcomes succumb to metastatic disease, local recurrence, or both. Local recurrence after resection occurs in $25 \%$ of patients with soft-tissue sarcomas, an event that portends eventual metastatic spread (1). Typically, the lung is the predominant site of metastasis, followed by bone and other soft-tissue locations. Prognostic factors include patient age, tumor grade (a risk assessment based on histopathologic characteristics), tumor depth (superficial or deep), tumor size, histologic subtype, surgical margin status at resection, and disease status (primary or recurrent disease) (1).

Prognostic factors adversely affecting the survival of patients with soft-tissue sarcomas are a deep tumor location, a tumor $5 \mathrm{~cm}$ or larger in greatest dimension, locally recurrent disease, a tumor located proximally in a lower extremity, and microscopic or grossly involved tumor margins at surgical resection (1). Sixty percent of adult soft-tissue sarcomas occur in the extremities. A tumor in a proximal lower extremity, a common location for softtissue sarcomas in adults, increases the risk for poor outcome because of its association with other poor risk factors.

TABLE 1

Common Subtypes of Soft-Tissue Sarcoma

\begin{tabular}{lc}
\hline \multicolumn{1}{c}{ Common subtype } & $\begin{array}{r}\text { Percentage in soft-tissue } \\
\text { sarcoma group (5) }\end{array}$ \\
\hline Leiomyosarcoma & 15 \\
Rhabdomyosarcoma & 4 \\
Alveolar (pediatric) & \\
Embryonal (pediatric) & \\
Pleomorphic (adult) & 24 \\
Fibrosarcoma & \\
Malignant fibrous & 20 \\
histiocytoma & \\
Liposarcoma & \\
Well differentiated & \\
Myxoid & \\
Round cell & \\
Pleomorphic & \\
Dedifferentiated & \\
Malignant peripheral nerve & \\
sheath tumor & \\
Malignant schwannoma & \\
Angiosarcoma & \\
Hemangiopericytoma & \\
Lymphangiosarcoma & \\
Pleomorphic sarcoma & \\
Synovial sarcoma & \\
Alveolar soft-part sarcoma & \\
GIST & \\
\hline
\end{tabular}

TABLE 2

Common Pediatric Sarcomas

Tumor histologic subtypes

Osteosarcoma
Chondroblastic
Fibroblastic
Osteoblastic
Parosteal
Telangiectatic (vascular)
Small cell
Periosteal
High-grade surface types
Secondary
Chondrosarcoma
Central, primary, secondary
Peripheral
Mesenchymal
Clear cell
Ewing sarcoma
Extraosseous Ewing sarcoma
Askin tumor (chest wall primary tumor)
Peripheral primitive neuroectodermal tumor/peripheral
neuroepithelioma
Giant cell tumor
Malignant giant cell tumor
Notochordal tumor (i.e., chordoma)

Poor risk factors include depth beneath the fascial plane and extensive tumor growth before diagnosis and treatment. In most patients, once metastases occur, survival time diminishes rapidly, despite salvage combination therapy regimens (4). Additionally, there are several treatment protocols for adults with sarcomas. Patients with low-grade small sarcomas are likely to proceed to tumor resection with the possibility of adjuvant radiation. Those with high-grade large tumors are often treated with doxorubicin-based neoadjuvant chemotherapy with or without radiation followed by resection and adjuvant therapy. Surgical resection ranges from simple excision with wide margins to complex limb salvage procedures.

Adult bone tumors are most often cartilaginous histologic types. Usually, only high-grade tumors are metastatic. Intermediate-grade and low-grade cartilage tumors have high morbidity rates due to repeated local recurrence and poor responses to neoadjuvant chemotherapy. Bone tumors are less frequent in older adults, and osteosarcomas and Ewing sarcomas are typically seen in younger adults. They rarely occur in adults older than $50 \mathrm{y}$. Bone tumors are metaphyseal tumors that spread primarily to the lungs. Risk factors for osteosarcoma include prior radiation therapy and germ line mutations (6). There are a few soft-tissue sarcoma histologic types that can also occur in bone, such as synovial sarcoma, a cancer that is more biologically consistent with its soft-tissue tumor counterparts. As in the soft-tissue sarcomas, prognostic factors include the presence of metastases at presentation and chemotherapy resistance. Treatment for bone sarcomas in adults is similar to that for soft-tissue sarcomas. Neoadjuvant doxorubicin-based 
chemotherapy is given and is followed by surgical resection and additional chemotherapy. Five-year survival depends largely on the level of chemotherapy resistance, which is approximately $60 \%$. The lower-grade osteosarcomas are treated with wide local excision and limb salvage procedures and typically recur in only about $5 \%$ of cases; however, these recurrences decrease 5-y survival significantly if the tissue recurrence degenerates into a higher grade or undifferentiated tumor type.

Pediatric sarcomas are dominated by osteosarcomas and Ewing sarcomas. Osteosarcomas have a peak incidence in patients who are in their second decade of life, and the age range begins at approximately the age of $10 \mathrm{y}$. Osteosarcomas predominately present in the extremities, and $15 \%-$ $20 \%$ of patients have pulmonary metastases at the time of diagnosis. In addition to prior radiation, the list of possible predisposing factors includes germ line p53 mutations, growth abnormalities, trauma, fetal x-ray exposure, and parental x-ray exposure. The presence of metastases at the time of diagnosis is prognostic of poor outcome. Treatment with intensive neoadjuvant chemotherapy results in a $60 \%-80 \% 5-y$ survival (6). Treatment response rate and overall survival rate are significantly higher for children with sarcomas than for adults with osteosarcomas.

Ewing sarcomas are also a bone tumor of adolescence, with a peak incidence around the age of $15 \mathrm{y}(6)$. They most commonly present as a painful mass in the extremities and more often are metastatic at presentation. Bone metastases (15\%-30\% of patients), lung metastases, and soft-tissue involvement adjacent to the primary tumor mass are common. Presumably, these tumors arise from the postsynaptic neural crest precursors and are synonymous with peripheral neuroectodermal tumors, which occur more predominantly in soft tissues (6). The presence of the EWS-FL1 fusion protein in the tumor confirms the tissue diagnosis. The Askin tumor, which occurs in the chest wall or lung, is a member of the Ewing sarcoma family of tumors and is also probably derived from the primitive neuroectoderm. There are no known risk factors for the development of these tumors, and they are rarely associated with other syndromes.

Patients with sarcoma have highly variable characteristics and clinical outcomes. Sarcomas are often multidrugresistant at initial diagnosis $(7,8)$. The multidrug resistance systems are tissue transporters that remove toxins from tissues and normally occur in tissue epithelial linings in the intestines, kidneys, and the blood-brain and placental barriers. The activity of multidrug resistance systems can be upregulated in sarcomas, and upregulation can be induced with chemotherapy agents that are substrates (911). This is a major cause of increased treatment failure and disease progression. The pathobiologic basis of multidrug resistance activity and other sarcoma characteristics is their complexity, which is likely a result of a substantial variety of genetic abnormalities. These consist of broad categories of tumor-specific translocations that contribute to tumor diagnostic criteria and sarcoma subtypes that have severe abnormalities in genetic and chromosomal instability (12). Sarcomas in almost all body locations present unique challenges for diagnosis and management. These challenges have presented opportunities for evaluation and validation of new imaging techniques.

\section{APPROACHES TO STAGING}

Because sarcomas are difficult to risk-stratify for outcome, staging systems have been devised to improve patient treatment and outcome. The American Joint Committee on Cancer criteria are often used clinically for staging soft-tissue sarcoma patients. This system undergoes periodic analyses and revisions. The current American Joint Committee on Cancer soft-tissue sarcoma system stratifies patient tumor by size $(\mathrm{T} 1 \leq 5 \mathrm{~cm}, \mathrm{~T} 2 \geq 5 \mathrm{~cm})$. Additionally, the tumor is subsequently characterized by histologic grade (I-III), the presence or absence of nodal and distant metastasis, and a special description of location with respect to fascial planes, which is denoted as either "a" or "b." The designation "a" denotes a superficial location, and "b" refers to deep tumor locations. Revisions that have been suggested include categories for very large tumors ( $\mathrm{T} 3 \geq 15 \mathrm{~cm}$ ), specific primary tumor sites, tumor margin statuses, histologic subtypes, the presence or absence of local tumor recurrences, and specific markers for biologic aggressiveness such as tumor necrosis.

The pathologic tumor grade is an important component of any tumor staging system; however, pitfalls exist in its application to individual patient tumors. The most widely used sarcoma grading system is that designed by the French Fédération Nationale des Centers de Lutte Contre le Cancer (13). Even with well-defined grading systems, there are several challenges in grading soft-tissue sarcomas. Tumors for which it is difficult to assign histologic grade because of rare characteristics include those in which the grade applied does not provide additional prognostic information beyond what is conferred from accurately determining the tumor histologic subtype. These include differentiated liposarcomas, the tumors that are considered "ungradable"; the epithelioid, clear cell, and angiosarcomas; and those for which histologic grade does not predict outcome well, such as malignant peripheral nerve sheath tumors (14). These findings reflect the heterogeneity of the biologic behavior of sarcomas and provide the stimulus for incorporation of newer systems that integrate tumor characteristics from several different sources that more accurately identify patient risk for tumor malignant behavior.

A comparative analysis of the soft-tissue sarcoma staging systems of the American Joint Committee on Cancer, the Memorial Sloan-Kettering Cancer Center, and the Musculoskeletal Tumor Society found that schemes that include tumor depth, grade, and size are most predictive of tumor relapse in patients with extremity tumors. Wunder et al. suggested that these systems can be used to identify patients who are most likely to benefit from participation in adjuvant therapy trials (15). 
Bone tumor staging follows the schemes used for other tumors with a few notable exceptions. The primary tumor is assessed by whether it is confined to the cortex (T1) or extends beyond this bone structure (T2) (16). Tumor histologic type and grade predominate among the staging criteria for prognosis. The presence or absence of distant metastases is also significant. As with soft-tissue sarcoma staging, bone sarcoma staging includes a careful examination of the lungs to discover metastases, which are often present at the time of initial diagnosis. Inclusion of nomograms for improved prognosis has also been developed, and their use may help to improve the selection of multimodality treatment for bone sarcoma patients (17).

Imaging plays an increasingly important role in sarcoma staging. All types of examinations-including plain films, chest radiographs, CT with and without contrast agent, MRI, and ${ }^{18}$ F-FDG PET—are now routinely used in combination to stage sarcoma patients and to restage patients when treatment decisions are recontemplated throughout different stages of diagnosis and during initial treatment. Plain films are useful as initial examinations for suspected bone tumors to begin characterization of the lesion. CT is useful for this purpose, with the additional benefit of the ability to determine the presence and extent of soft-tissue involvement. In soft-tissue sarcomas, CT is the standard for characterizing the tumor mass. More recently, MRI has been used to help define tumor components and provide fine detail on involvement of surrounding structures, particularly in the extremities. Although ${ }^{201} \mathrm{Tl}$ has been used, the specificity of ${ }^{18}$ F-FDG PET for sarcoma provides a more complete and biologically relevant tumor image. The bone scan is still routinely used because of its sensitivity in detection of bone metastases, although most sarcomas predominantly metastasize to the lungs. Several types commonly have associated bone metastases, such as osteosarcoma, Ewing sarcoma, and highly undifferentiated tumors. Imaging methods and their uses in sarcoma management are discussed more thoroughly in the following sections.

\section{RADIOPHARMACEUTICALS}

Disease staging in sarcoma involves all aspects of imaging the body with radiopharmaceuticals. Because these tumors vary considerably in their presentation, local recurrence rates, and patterns of metastatic spread, different imaging studies that provide complementary information for disease staging are used in clinical practice. At this time, there is no strong consensus on which imaging modalities are most important in diagnosis and treatment monitoring in sarcoma. MRI, PET, CT, bone scanning, and plain radiography all contribute complementary information that helps in patient management. These are for characterizing extent of tumor and the presence of bony, lymph node, and soft-tissue metastases. Some tumor types involve multifocal primary disease presentation, synchronous tumors, and skip metastases. In most sarcoma clinics, a combination of nonspecific, highly sensitive, and tissue-specific imaging agents is used in diagnosis, staging, response, termination, and restaging aspects of patient management.

The standard ${ }^{99 \mathrm{~m}} \mathrm{Tc}-\mathrm{methylene}$ diphosphonate (MDP) bone scan is widely used in oncology for staging the skeleton for the presence of bony metastases. Sarcomas generally show osteoblastic behavior and are less likely to show lytic metastases. Often, as in the case of bone tumors, they show either a blastic or lytic appearance. For soft-tissue masses, the bone scan is a standard part of disease staging. Patients with bony metastases show increased uptake in typical metastatic disease patterns in the axial skeleton and extremities. The presence or absence of uptake is often diagnostic in separating more benign or locally aggressive tumors from malignant types. Bone scans also have special utility in primary bone tumors. These tumors form malignant osteoid matrices that are undergoing disordered calcification; therefore, they are ${ }^{99 \mathrm{~m}} \mathrm{Tc}-\mathrm{MDP}-$ avid (Fig. 1). Both the osteosarcomas and some chondrosarcomas with osteoblastic differentiation contain malignant osteoid. Ewing sarcomas cause bone destruction and reactions that result in bone scan positivity. Primary bone tumors have frequent bone metastases and can present with skip lesions in the affected bone. The osteosarcomas frequently have bone metastases, but a bone scan-positive appearance in multiple sites is common in Ewing sarcoma. In this latter group of tumors, metastases are often present at the time of diagnosis in both bone and soft-tissue (peripheral neuroectodermal) primary tumors.

The bone scan is also a sensitive method for indentifying osteosarcoma pulmonary metastases. These are often highly ${ }^{99 \mathrm{~m} T c-M D P-a v i d .}$ Some clinicians use the relative decrease in bone scan uptake in pulmonary metastases as an indicator of treatment response and suitability for wedge resection metastasectomy. In osteosarcoma patients with limited pulmonary metastasis, this procedure has been shown to increase disease-free survival (18). The ${ }^{99 \mathrm{~m} T c-M D P}$ scan has been reported to contribute to the diagnosis of boneforming malignant tumors by revealing unusual sites and presentations. As such, in these individuals, similar to primary bone tumor patients, the ${ }^{99 \mathrm{~m} T c-M D P}$ bone scan is likely a means of monitoring treatment response. In these patients, a decrease in malignant osteoid matrix calcification would indicate a response to treatment in this cellular portion of the tumor. The bone scan appearance in metastatic disease, as with other tumors, can also underestimate the extent and severity of skeletal metastases. In these cases, the metastases involving the bone may be apparent only when a cortical reaction occurs. Subsequent other whole-body surveys such as $\mathrm{CT}$ or ${ }^{18} \mathrm{~F}-\mathrm{FDG}$ PET may provide a complementary picture of the extent of diseased skeleton (19).

${ }^{201}$ Tl-chloride as a nonspecific tumor imaging agent has been described for use in many tumors, including sarcoma. Most often, higher uptake is noted in malignant tumors, whereas lower uptake more frequently signifies benign neoplasms. Soft-tissue, bone, and cartilaginous tumors have shown positivity (20). Typical high-grade tumor features, 


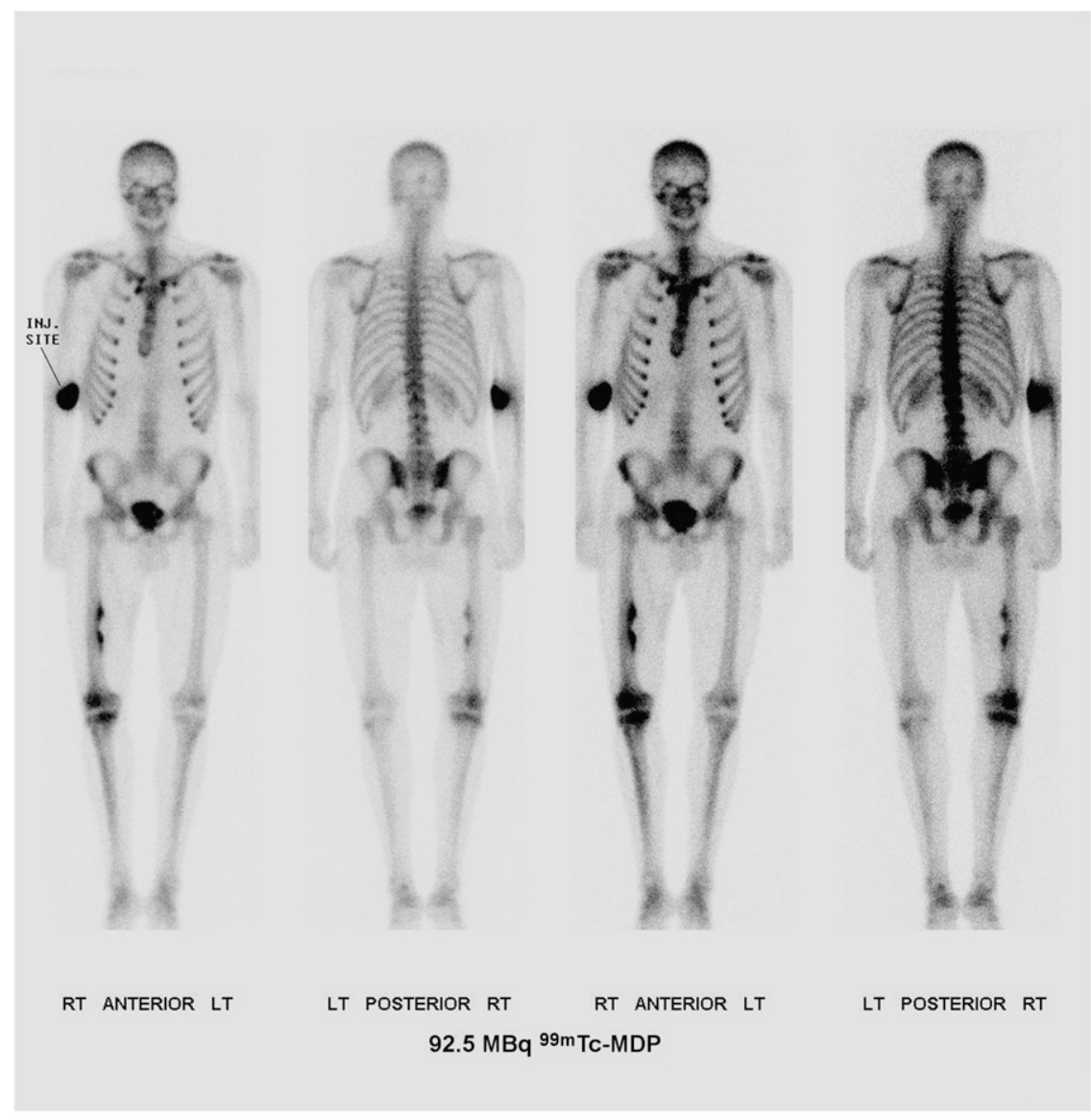

FIGURE 1. 99mTc-MDP anterior and posterior whole-body planar images of patient with osteosarcoma in mid left femur. such as central necrosis, can also be noted in ${ }^{201} \mathrm{Tl}$-chloride imaging; this finding is predictive of decreased survival (21).

${ }^{18} \mathrm{~F}$-fluoride is increasingly being used as a sensitive bone scanning agent for metastatic surveys for many cancers. This is also the case for sarcomas; however, the use of ${ }^{18}$ F-fluoride may extend beyond detection of metastases in bone sarcomas. Because the hallmark of these tumors is new bone formation, uptake and distribution levels of ${ }^{18}$ F-fluoride will likely change in response to therapy. A particular use may be in evaluating activity in lung metastases because it is common practice to resect lung metastases when they are quiescent after successful chemotherapy.

\section{${ }^{18}$ F-FDG AND SARCOMA DIAGNOSIS}

${ }^{18} \mathrm{~F}-\mathrm{FDG}$ PET has been evaluated for use in sarcoma imaging. Recently, the use of this imaging procedure in treatment response evaluation has been investigated. Diagnosis through imaging in primary cancers has several critical aspects for patient treatment planning. Imaging is used to determine the biologic behavior of the tumor, often determining if the tumor is benign or malignant; however, sarcomas include numerous tumors that do not fit well with either category because they are locally aggressive yet rarely meta- static. The tumor histologic type determined from biopsy and imaging data is also helpful in determining tumor grade, which is the propensity of the tumor to behave aggressively. Several prospective and retrospective studies have described the utility of ${ }^{18} \mathrm{~F}$-FDG PET in sarcoma diagnosis. In softtissue sarcoma, this imaging can reliably distinguish lowgrade from high-grade tumors (22-25). Low-grade tumors are often difficult to distinguish through histologic criteria as well. Additionally, special features and ${ }^{18} \mathrm{~F}-\mathrm{FDG}$ uptake are related to specific histologic types. For example, the atypical lipomas and the low-grade liposarcomas may have similar tissue features and biologic behavior. They often result in repeated local recurrence; nevertheless, in a large mass, the ${ }^{18}$ F-FDG appearance can be significant for identifying small areas of high-grade differentiation, which can lead to a much worse treatment outcome than the low-grade majority of the tumor mass composition. Figure 2 shows ${ }^{18}$ F-FDG PET examples of primary sarcoma tumors.

The ${ }^{18}$ F-FDG image can be helpful in identifying areas for diagnostic biopsy for similar reasons. The soft-tissue sarcomas can be highly heterogeneous in their histologic characteristics, which often confer heterogeneity in ${ }^{18} \mathrm{~F}$ FDG spatial uptake distribution. These unique tumor features may account for high levels of treatment resistance and variable responses to multimodality therapy. Spatial heterogeneity in sarcoma uptake has been shown to be 
A

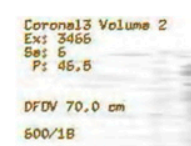

FIGURE 2. ${ }^{18} \mathrm{~F}-\mathrm{FDG}$ PET images of patients with primary sarcoma tumors. (A) Coronal image of patient with leiomyosarcoma of bone in left femur. (B) Coronal image of patient with angiosarcoma in right pelvis.

\section{B}

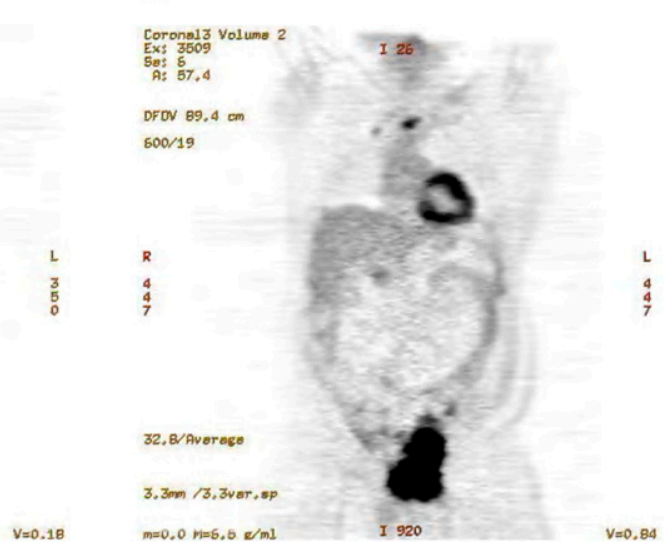

independently predictive of patient outcome. Eary et al. found that baseline ${ }^{18} \mathrm{~F}-\mathrm{FDG}$ in diagnostic scans and tumor grade was somewhat weaker in outcome prediction, suggesting that new methods for incorporating ${ }^{18} \mathrm{~F}-\mathrm{FDG}$ tumor uptake distribution and for incorporating standardized uptake value into diagnostic methods for sarcomas can provide significant tumor diagnostic information (26). Figure 3 shows tumors with high levels of ${ }^{18} \mathrm{~F}$ FDG uptake heterogeneity.

As for other tumors, ${ }^{18} \mathrm{~F}-\mathrm{FDG}$ PET has been used for effective tumor staging. It can be used to identify bone and soft-tissue metastases and nodal metastases in those types where they occur (epithelioid and synovial sarcomas). Although lung metastases are the most common site of metastases in patients with high-grade tumors, these are often small and are not visualized on ${ }^{18}$ F-FDG PET. For this reason, a complete staging examination for both softtissue and bony sarcomas also consists of a high-resolution contrast-enhanced CT scan of the chest.

\section{SARCOMA TREATMENT}

Soft-tissue sarcoma treatment plans are determined primarily by tumor grade. Large intermediate-grade and high-grade sarcomas often receive neoadjuvant adriamycinbased chemotherapy with or without preoperative radiation. After resection, if a response was obtained based on percentage tumor necrosis in the resected tumor, adjuvant therapy is started. If an adequate response was not obtained, an alternative chemotherapy regimen usually replaces the ineffective treatment. Bone tumors are also treated through clinical protocols that have varying durations for neoadjuvant treatment, depending on patient age. Pediatric patients receive the most intense therapy, which yields good long-term outcome rates. Ewing sarcoma patients also participate in protocol studies, and bone marrow transplantation is an option that is considered in some advanced cases. Molecular imaging plays an increasingly important role in treatment planning and response assessment.

\section{F-FDG IN SARCOMA RESTAGING AND TREATMENT RESPONSE}

A review of cancer treatment response for sarcoma as with other cancers begins with consideration of the pathologic basis of the tumor process. Sarcomas are complex pathobiologic processes that may exhibit a wide range of blood flow, cell proliferation rate, cell viability,
FIGURE 3. Heterogenous ${ }^{18} \mathrm{~F}-\mathrm{FDG}$ uptake in high-grade sarcomas. (A) Coronal image of patient with malignant peripheral nerve sheath tumor in right thigh. (B) Coronal image of patient with high-grade pleomorphic sarcoma not otherwise specified in right thigh.
A

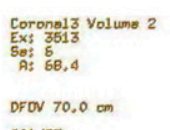

DFON $70.0 \mathrm{~cm}$
$601 / 23$

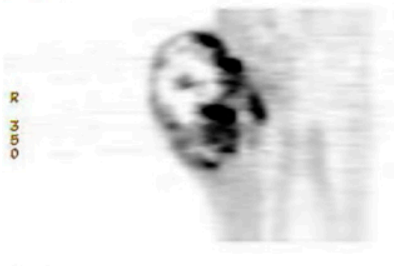

5.5

$3.3 \mathrm{~mm} / 3.3 \mathrm{var}, \mathrm{sp}$

$m=0.0 \mathrm{m=5.4} \approx \mathrm{ml}$

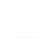

(2)

5.5

$3.3 \mathrm{~mm} / 3,3 \mathrm{ver}, \mathrm{sp}$

$v=1.61$
B

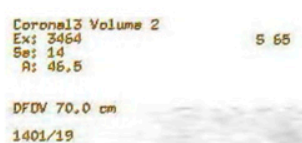

DFOV 70.0
$1401 / 19$

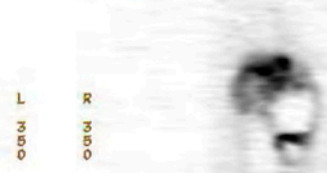

$m=0.00 \mathrm{~m}=10,00 \mathrm{e} / \mathrm{m}$
I 635

Ves. 82 
inflammation, $\mathrm{pH}$, oxygenation, and numerous other processes. Because of these processes, treatment response can differ significantly from a standard treatment combination or from treatments with different mechanisms of action. The ability of ${ }^{18}$ F-FDG PET to identify treatment response is an important goal for the sarcoma patient population. These tumors often do not change size in response to neoadjuvant chemotherapy because they can comprise tissue elements that do not change in tumor response, or they undergo slow changes in size reduction, such as in bone, cartilage, scar, and myxoid areas. Consequently, the RECIST (Response Evaluation Criteria in Solid Tumors) criteria for treatment response do not apply well to this group, and ${ }^{18} \mathrm{~F}-\mathrm{FDG}$ has an advantage, as described by Evelevitch (27). In that work, a $60 \%$ decrease in tumor ${ }^{18} \mathrm{~F}-\mathrm{FDG}$ uptake compared with baseline had a sensitivity of $100 \%$ and a specificity of $71 \%$ for histologic response, whereas RECIST criteria for response applied to the same group showed a sensitivity of $25 \%$ and a specificity of $100 \%(28,29)$. In sarcoma clinical practice, tumor treatment response assessments must provide information on the nature and timing of response. Patients with large intermediate-grade and high-grade tumors receive neoadjuvant chemotherapy and possibly radiotherapy. If a clinical imaging and histopathologic response is observed, then similar adjuvant treatment will be continued; however, if responses are not observed, then second-line or experimental therapies will be considered because the patient is highly likely to be at an even higher risk for metastases and shortened survival. Because clinical and histopathologic response evaluations can be somewhat subjective, imaging tumor response quantitatively can provide clinically relevant objective information for treatment planning for a sarcoma patient. For sarcoma patients for whom treatment choices are limited and often highly toxic, newer therapies that are directed at specific molecular targets may be cytostatic and result in tumor growth arrest, which can be observed effectively with ${ }^{18} \mathrm{~F}$-FDG PET. These changes may indicate effective therapy for a patient, as opposed to direct cell-killing mechanisms and tumor shrinkage, and they may indicate improved patient outcome.

Tumor cellular necrosis fraction is considered the hallmark of treatment response to chemotherapy in sarcomas; however, overinterpretation of tumor cellular necrosis in a tumor specimen may result in cases for which necrosis was present as a distinguishing feature of the primary tumor. Figure 4 shows examples of primary sarcomas in which significant necrosis was present before therapy. For this reason, reliable treatment response imaging in sarcoma requires a baseline pretreatment scan for comparison. Necrosis can take the form of coagulative necrosis or hemorrhagic necrosis when response results in hemorrhage and its resolution. Scarring is a common treatment response and is also common in radiation treatment. Compared with necrosis, scarring as a treatment response is metabolically active and can cause significant ${ }^{18}$ F-FDG uptake. When granulation tissue formation precedes scarring, the inflammatory cells present may also elevate the apparent tumor bed tissue metabolism. Activated white cells can show as much as a 10 -fold difference in ${ }^{18} \mathrm{~F}$-FDG uptake, complicating image interpretation. An important part of treatment response interpretation in sarcoma is identifying the treatment agent mechanism, tumor subtype, and timing of the scan observation in relation to the course and type of treatment. Early after therapy, observations may reveal very different findings from those obtained after the biologic mechanisms involved in treatment response have reached a more static state. Radiation responses to tumor and surrounding tissues may have very different timescales in relation to the end of the final therapy response. In fact, early detection of treatment response that indicates improved patient outcome with newer therapies is an area of active research. The ability of ${ }^{18} \mathrm{~F}$-FDG PET to identify treatment response is an important goal for the sarcoma patient population.

Many groups advocate the use of the ${ }^{18} \mathrm{~F}-\mathrm{FDG}$ PET tumor standardized uptake value to measure sarcoma uptake and to monitor treatment response. A 35\% reduction
A

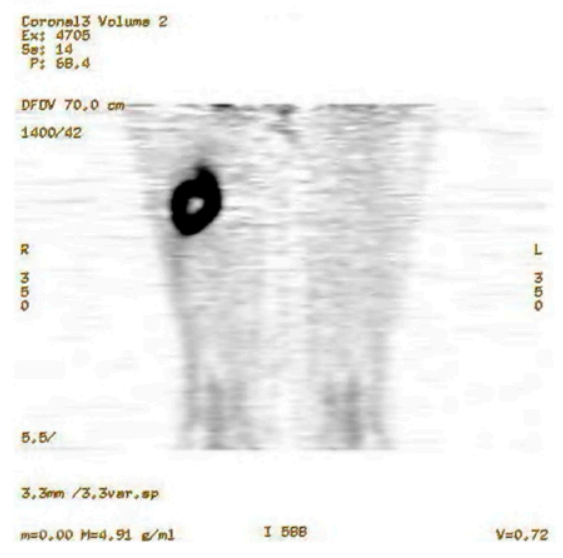

B

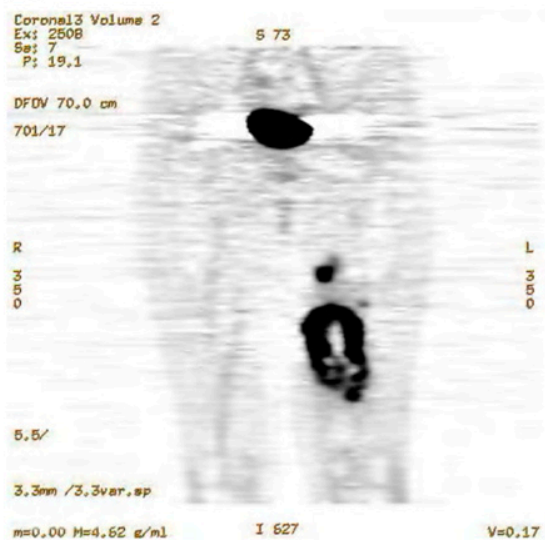

FIGURE 4. Sarcomas showing central necrosis, a feature of high-grade behavior. (A) Coronal image of patient with high-grade sarcoma, not otherwise specified, in right thigh. (B) Coronal image of patient with osteosarcoma in left femur. 
in tumor ${ }^{18} \mathrm{~F}-\mathrm{FDG}$ uptake from baseline is predictive of histologic response $(27,28,30)$. If the ${ }^{18} \mathrm{~F}-\mathrm{FDG}$ PET study is performed in a standard, consistent manner, the standardized uptake value is a robust value for comparison of one imaging study to another in the same patient at a later time and in patients in different groups. The optimum parameters for ${ }^{18} \mathrm{~F}$-FDG imaging in cancer were recently described in a report of a National Cancer Institute consensus committee, where standard techniques for the use of ${ }^{18} \mathrm{~F}-\mathrm{FDG}$ as a biomarker for cancer treatment were presented (31). Use of ${ }^{18}$ F-FDG PET as a biomarker or surrogate endpoint for patient outcome is the basis for clinical research studies that determine the sensitivity and specificity of the method for following response to treatment and for assessing normal-tissue damage as a result of treatment. Few effective treatment strategies exist for sarcoma, yet there is a great potential for new therapy strategies. ${ }^{18}$ F-FDG PET can be a powerful tool for evaluation of the effectiveness of novel treatments $(32-36)$.

The most dramatic example of ${ }^{18}$ F-FDG PET as a biomarker for treatment response assessment is in gastrointestinal stromal tumors (GIST) treated with imatinib mesylate. GIST are ${ }^{18}$ F-FDG-avid and can yield impressive PET images. Goerres et al. found that median survival of patients who demonstrated an ${ }^{18}$ F-FDG PET response was $100 \%$ at $2 \mathrm{y}$, compared with a group with residual tumor uptake after treatment. The study also demonstrated ability to separate patients by time to tumor progression based on levels of ${ }^{18} \mathrm{~F}$-FDG uptake in tumors (37). Early response to imatinib mesylate in the GIST population detected by ${ }^{18} \mathrm{~F}$ FDG PET has also been shown. As much as a $65 \%$ decrease in tumor ${ }^{18} \mathrm{~F}-\mathrm{FDG}$ uptake was demonstrated at the end of 1 wk of effective therapy, and as high as a 95\% response detected by 1 mo after treatment initiation has been found by other groups. Response detection using CT criteria was less accurate, including no significant $\mathrm{CT}$ responses noted in ${ }^{18} \mathrm{~F}-\mathrm{FDG}$ PET-responsive patients (38-43). ${ }^{18} \mathrm{~F}-\mathrm{FDG}$ PET of GIST patients at baseline is recommended to observe maximum tumor activity levels and to allow accurate staging. Repeated imaging is suggested in the first month after therapy initiation to observe response and to predict treatment effect. Another imaging examination may be helpful if treatment resistance is suspected and if there is a need to establish a new baseline of tumor uptake and location for treatment observation. ${ }^{18} \mathrm{~F}-\mathrm{FDG}$ imaging for GIST treatment response evaluation has been incorporated into the guidelines for GIST management determined by an international consensus conference (44).

Treatment response in other sarcomas using ${ }^{18} \mathrm{~F}-\mathrm{FDG}$ PET has been demonstrated in several studies. Soft-tissue sarcomas represent most of the sarcomas that occur in adults. Treatment response imaging for this group of patients is emerging (45-49). During an early study, in an extremity soft-tissue sarcoma group treated with adriamycin-based neoadjuvant chemotherapy, Schuetze et al. showed that separating patients by their ${ }^{18} \mathrm{~F}-\mathrm{FDG}$ response $(>40 \%)$ showed a significant difference in survival for each group (50). Patients in the ${ }^{18} \mathrm{~F}-\mathrm{FDG}$ PET nonresponse group had a $90 \%$ risk of disease recurrence at $4 \mathrm{y}$, compared with ${ }^{18}$ F-FDG responders. These data, and those of others, may indicate the effectiveness and survival increase in softtissue sarcoma patients treated with neoadjuvant chemotherapy before tumor resection. Figure 5 shows examples of treatment response documented by ${ }^{18} \mathrm{~F}$-FDG PET for a leiomyosarcoma and a Ewing sarcoma. This imaging technique may be useful for patients who have tumor resistance during the course of therapy and, therefore, might benefit from treatment intensification or early resection.

A similar finding in the Ewing sarcoma population has been shown by Hawkins et al. (51). Patients whose tumors showed increased standardized uptake value ratios between the baseline and preresection (after neoadjuvant chemotherapy) scans had significantly improved survival. In this patient group, in the future, good responders may be identified for less toxic treatment protocols. Studies in imaging osteosarcoma for treatment response have also been conducted. Early studies by Schulte et al. showed that changes in tumor ${ }^{18} \mathrm{~F}-\mathrm{FDG}$ uptake correlated with tumor necrosis levels in patients treated with neoadjuvant chemotherapy. Implications for limb salvage surgery were described because such complex tumor resection procedures might not be considered in nonresponders (52). Others have recently shown similar results for ${ }^{18} \mathrm{~F}-\mathrm{FDG}$ imaging in osteosarcoma $(53,54)$.

Pediatric sarcomas are usually bony tumors, but they have been considered in several studies because the treatment for pediatric patients somewhat differs from protocols for adults. Similar to their findings for Ewing sarcomas in this mixed group of osteosarcoma and Ewing sarcoma patients, Hawkins et al. found that the ratios of ${ }^{18} \mathrm{~F}-\mathrm{FDG}$ uptake between baseline and postneoadjuvant therapy completion correlated with histologic response (55). Noting a need for prospective imaging studies to be conducted on pediatric sarcoma populations, both Franzius et al. and McCarville et al. published data on small pediatric sarcoma subgroups to demonstrate the clinical utility of ${ }^{18} \mathrm{~F}-\mathrm{FDG}$ imaging $(56,57)$. The latter group included a rhabdomyosarcoma subgroup, a tumor subtype for which most patients are treated under cooperative group therapy protocols. A correlation was also noted between longer survival and ${ }^{18}$ F-FDG changes in response to therapy in a small retrospective study of patients with rhabdomyosarcoma. This underscored the difficulty of performing prospective studies on pediatric tumor groups, in which patients with these serious tumors are infrequent.

${ }^{18} \mathrm{~F}-\mathrm{FDG}$ imaging in treatment response can significantly contribute to the care of sarcoma patients by identifying clinically relevant responses, or a lack thereof, and in providing predictive information for patient outcome. Although sarcomas are a less frequent form of malignancy in the population, they affect the entire human age span, and they constitute a 
A

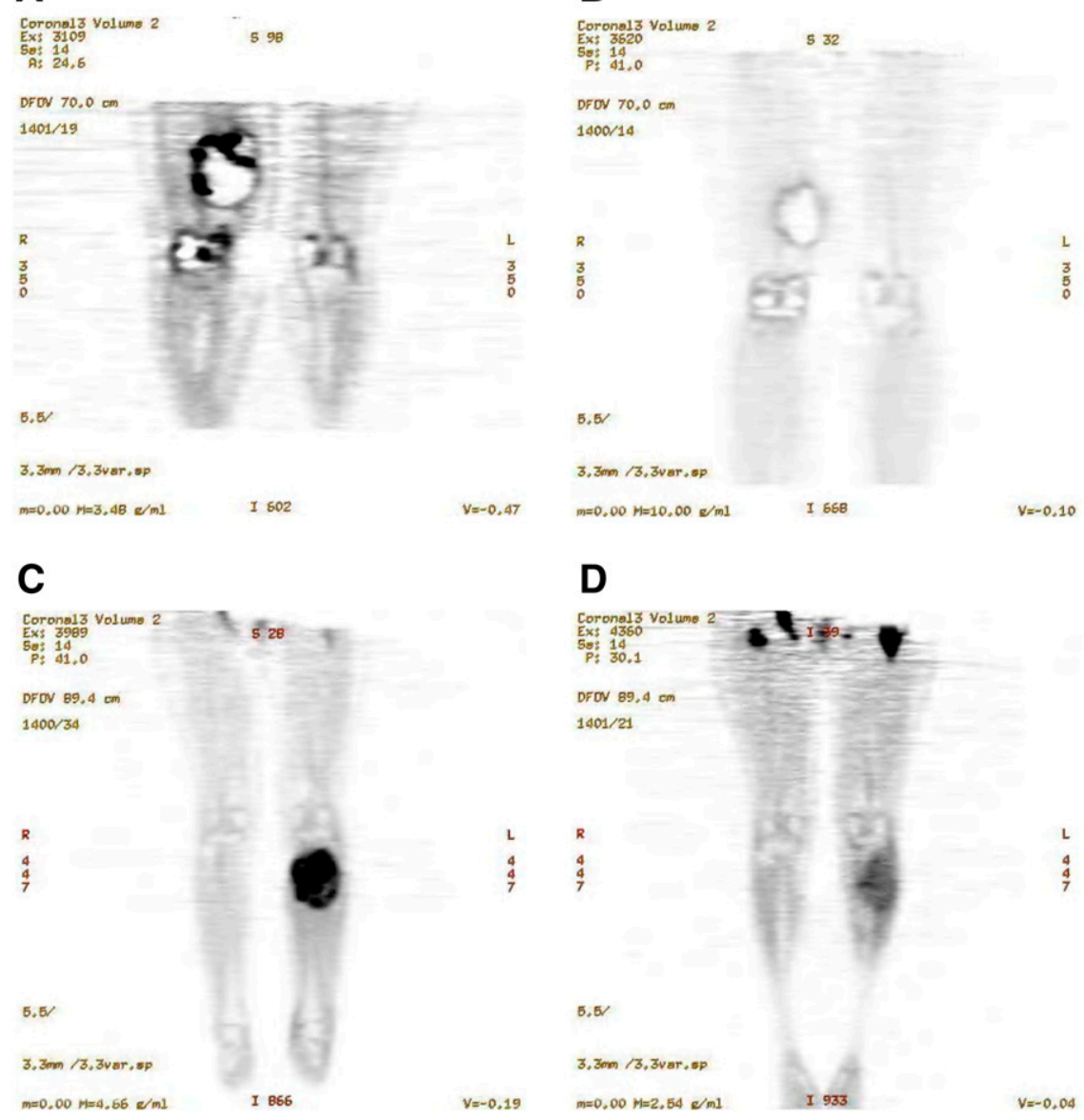

B

$\mathrm{L}$
5
5

FIGURE 5. ${ }^{18}$ F-FDG PET examples of neoadjuvant treatment response in sarcomas. (A) Coronal image of patient with leiomyosarcoma in right thigh. (B) Same patient as in $\mathrm{A}$, showing decrease in tumor ${ }^{18} \mathrm{~F}-\mathrm{FDG}$ uptake after treatment. (C) Coronal image of patient with Ewing sarcoma in left leg. (D) Same patient as in C, showing decrease in tumor ${ }^{18} \mathrm{~F}-\mathrm{FDG}$ uptake after treatment. large number of affected individuals in aggregate. Currently, treatment for the more aggressive high-grade tumors and for locally recurrent low-grade tumors often results in less than optimum treatment outcomes. Future clinical trials of newer treatment combinations for sarcoma patients will benefit from incorporating ${ }^{18} \mathrm{~F}$-FDG PET treatment response information, as has been true for patients with other cancer types. For the individual patient, ${ }^{18} \mathrm{~F}$-FDG PET can provide critical information about treatment response and indicate further treatment planning.

\section{CURRENT STUDIES AND THE FUTURE OF NEW IMAGING IN SARCOMA}

Other PET radiopharmaceuticals that are more specific than ${ }^{18} \mathrm{~F}$-FDG for biologically relevant aspects of sarcomas are under investigation. Measurement of levels of tumor hypoxia has clinical significance for many tumors, including sarcoma $(58,59)$. Hypoxia is known to confer resistance to both radiotherapy and chemotherapy in sarcomas. Most sarcomas have some level of hypoxia, which confers resistance to both radiotherapies and chemotherapies. Newer therapies that target hypoxic tumor tissues after identification of these regions may improve patient outcome. PET radiopharmaceuticals designed to quantify tumor prolifer- ation will also play an important role in the diagnosis and treatment assessment of sarcomas $(60,61)$. $3^{\prime}$-deoxy- $3^{\prime}-{ }^{18} \mathrm{~F}-$ fluorothymidine $\left({ }^{18} \mathrm{~F}\right.$-FLT) is a thymidine analog that has been examined in a few sarcoma imaging trials. Tumor uptake in bone and soft-tissue tumors correlated with tumor grade and proliferating cell fraction determined on histologic examination $(62,63)$. Been et al. in 2007 showed that ${ }^{18}$ F-FLT tumor uptake decreased significantly with treatment and that tumors with initially high ${ }^{18}$ F-FLT uptake showed greater treatment responses (64). These findings suggest that ${ }^{18} \mathrm{~F}$-FLT may play a complementary role as a therapy biomarker for prediction and identification of tumor response. Because sarcomas are a highly variable group of tumors, with similarly variable clinical outcomes, their cellular proliferation levels likely indicate increased risk of metastasis and poor clinical outcome. Newer molecularly targeted therapies are currently in clinical trials for patients with tumors resistant to standard chemotherapy. These newer therapies will likely have early changes in cellular proliferation in response to effective therapy. Sarcoma patients are likely to benefit from these more biologically specific imaging techniques that may provide insight regarding treatment effectiveness. Molecular imaging research in PET is being directed toward identifying tumor characteristics that 
can be used to define patient risk for aggressive disease and for noninvasive treatment monitoring.

\section{ACKNOWLEDGMENTS}

This work was supported by NIH NCI 65537, NIH P01 CA042045, and NCRR S10 RR17229.

\section{REFERENCES}

1. Pisters PW, Leung DH, Woodruff J, Shi W, Brennan MF. Analysis of prognostic factors in 1,041 patients with localized soft tissue sarcomas of the extremities. $J$ Clin Oncol. 1996;14:1679-1689.

2. Gadgeel SM, Harlan LC, Zeruto CA, Osswald M, Schwartz AG. Patterns of care in a population-based sample of soft tissue sarcoma patients in the United States. Cancer. 2009;115:2744-2754.

3. Salas S, Stoeckle E, Collin F, et al. Superficial soft tissue sarcomas (S-STS): a study of 367 patients from the French Sarcoma Group (FSG) database. Eur J Cancer. 2009;45:2091-2102.

4. Engellau J, Bendahl PO, Persson A, et al. Improved prognostication in soft tissue sarcoma: independent information from vascular invasion, necrosis, growth pattern, and immunostaining using whole-tumor sections and tissue microarrays. Hum Pathol. 2005;36:994-1002.

5. Nair SG, Amadio CH, Scarlato M, Forman DA. Frequency of histopathologic types of soft tissue sarcoma (STS) reported from a large community-based hospital over a 20 year period [abstract]. J Clin Oncol. 2006;24(suppl):19507.

6. Eary JF. PET-CT and SPECT-CT of malignant bone tumors and PET and PET-CT in soft tissue sarcomas. In: von Schulthess GK, ed. Molecular Anatomic Imaging: PET-CT and SPECT-CT Integrated Modality Imaging. 2nd ed. Philadelphia, PA: Lippincott Williams \& Wilkins; 2007:443-449, 456-467.

7. Coley HM, Verrill MW, Gregson SE, Odell DE, Fisher C, Judson IR. Incidence of P-glycoprotein overexpression and multidrug resistance (MDR) reversal in adult soft tissue sarcoma. Eur J Cancer. 2000;36:881-888.

8. Komdeur R, Plaat BE, Hoekstra HJ, et al. Expression of P-glycoprotein, multidrug resistance-associated protein 1 , and lung resistance-related protein in human soft tissue sarcomas before and after hyperthermic isolated limb perfusion with tumor necrosis factor-alpha and melphalan. Cancer. 2001;91:1940-1948.

9. Komdeur R, Plaat BE, van der Graaf WT, et al. Expression of multidrug resistance proteins, P-gp, MRP1 and LRP, in soft tissue sarcomas analysed according to their histological type and grade. Eur J Cancer. 2003;39:909-916.

10. Komdeur R, Molenaar WM, Zwart N, Hoekstra HJ, van den Berg E, van der Graaf WT. Multidrug resistance proteins in primary and metastatic soft-tissue sarcomas: down-regulation of P-glycoprotein during metastatic progression. Anticancer Res. 2004;24:291-295.

11. Oda Y, Saito T, Tateishi N, et al. ATP-binding cassette superfamily transporter gene expression in human soft tissue sarcomas. Int J Cancer. 2005;114:854-862.

12. Helman LJ, Meltzer P. Mechanisms of sarcoma development. Nat Rev Cancer. 2003;3:685-694.

13. Coindre JM, Terrier P, Guillou L, et al. Predictive value of grade for metastasis development in the main histologic types of adult soft tissue sarcomas: a study of 1240 patients from the French Federation of Cancer Centers Sarcoma Group. Cancer. 2001;91:1914-1926.

14. Deyrup AT, Weiss SW. Grading of soft tissue sarcomas: the challenge of providing precise information in an imprecise world. Histopathology. 2006;48: $42-50$.

15. Wunder JS, Healey JH, Davis AM, Brennan MF. A comparison of staging systems for localized extremity soft tissue sarcoma. Cancer. 2000;88:2721-2730.

16. Spiessl B, Beahrs OH, Hermanek P, et al. eds. TNM Atlas: Illustrated Guide to the TNM/pTNM Classification of Malignant Tumours. 3rd ed, 2nd rev. Berlin, Germany: Springer-Verlag; 1992.

17. Kim MS, Lee SY, Lee TR, et al. Prognostic nomogram for predicting the 5-year probability of developing metastasis after neo-adjuvant chemotherapy and definitive surgery for AJCC stage II extremity osteosarcoma. Ann Oncol. 2009; 20:955-960.

18. Antunes M, Bernardo J, Salete M, Prieto D, Eugénio L, Tavares P. Excision of pulmonary metastases of osteogenic sarcoma of the limbs. Eur J Cardiothorac Surg. 1999;15:592-596.

19. Iagaru A, Goris ML. Rhabdomyosarcoma diffusely metastatic to the bone marrow: suspicious findings on ${ }^{99} \mathrm{mTc}-\mathrm{MDP}$ bone scintigraphy confirmed by ${ }^{18} \mathrm{~F}-18 \mathrm{FDG}$ PET/ CT and bone marrow biopsy. Eur J Nucl Med Mol Imaging. 2008;35:1746.
20. Goto Y, Ihara K, Kawauchi S, Ohi R, Sasaki K, Kawai S. Clinical significance of thallium-201 scintigraphy in bone and soft tissue tumors. J Orthop Sci. 2002; 7:304-312.

21. McCarville MB, Barton EH, Cameron JR, et al. The cause and clinical significance of central tumor photopenia on thallium scintigraphy of pediatric osteosarcoma of the extremity. AJR. 2007;188:572-578.

22. Eary JF, Conrad EU, Bruckner JD, et al. Quantitative [F-18]fluorodeoxyglucose positron emission tomography in pretreatment and grading of sarcoma. Clin Cancer Res. 1998;4:1215-1220.

23. Nieweg OE, Pruim J, van Ginkel RJ, et al. Fluorine-18-fluorodeoxyglucose PET imaging of soft-tissue sarcoma. J Nucl Med. 1996;37:257-261.

24. Griffeth LK, Dehdashti F, McGuire AH, et al. PET evaluation of soft-tissue masses with fluorine-18 fluoro-2-deoxy-D-glucose. Radiology. 1992;182:185-194.

25. Ioannidis JP, Lau J. 18-FDG PET for the diagnosis and grading of soft-tissue sarcoma: a meta-analysis. J Nucl Med. 2003;44:717-724.

26. Eary JF, O'Sullivan F, O'Sullivan J, Conrad EU. Spatial heterogeneity in sarcoma ${ }^{18} \mathrm{~F}-$ FDG uptake as a predictor of patient outcome. J Nucl Med. 2008;49:1973-1979.

27. Evilevitch V, Weber WA, Tap WD, et al. Reduction of glucose metabolic activity is more accurate than change in size at predicting histopathologic response to neoadjuvant therapy in high-grade soft-tissue sarcomas. Clin Cancer Res. 2008;14:715-720.

28. Benz MR, Czernin J, Tap WD, et al. FDG-PET/CT imaging predicts histopathologic treatment responses after neoadjuvant therapy in adult primary bone sarcomas. Sarcoma. April 18, 2010 [Epub ahead of print].

29. Benjamin RS, Choi H, Macapinlac HA, et al. We should desist using RECIST, at least in GIST. J Clin Oncol. 2007;25:1760-1764.

30. Schuetze SM, Baker LH, Benjamin RS, Canetta R. Selection of response criteria for clinical trials of sarcoma treatment. Oncologist. 2008;13(suppl 2):32-40.

31. Shankar LK, Hoffman JM, Bacharach S, et al. Consensus recommendations for the use of ${ }^{18} \mathrm{~F}$-FDG PET as an indicator of therapeutic response in patients in National Cancer Institute Trials. J Nucl Med. 2006;47:1059-1066.

32. Kelloff GJ, Hoffman JM, Johnson B, et al. Progress and promise of FDG-PET imaging for cancer patient management and oncologic drug development. Clin Cancer Res. 2005;11:2785-2808.

33. Benz MR, Allen-Auerbach MS, Eilber FC, et al. Combined assessment of metabolic and volumetric changes for assessment of tumor response in patients with soft-tissue sarcomas. J Nucl Med. 2008;49:1579-1584.

34. Weber WA. Use of PET for monitoring cancer therapy and for predicting outcome. J Nucl Med. 2005;46:983-995.

35. Jerusalem G, Belhocine TZ. Metabolic monitoring of chemosensitivity with ${ }^{18}$ FDG PET. Methods Mol Med. 2005;111:417-440.

36. Larson SM, Erdi Y, Akhurst T, et al. Tumor treatment response based on visual and quantitative changes in global tumor glycolysis using PET-FDG imaging: the visual response score and the change in total lesion glycolysis. Clin Positron Imaging. 1999;2:159-171.

37. Goerres GW, Stupp R, Barghouth G, et al. The value of PET, CT and in-line PET/ $\mathrm{CT}$ in patients with gastrointestinal stromal tumours: long-term outcome of treatment with imatinib mesylate. Eur J Nucl Med Mol Imaging. 2005;32:153-162.

38. Stroobants S, Goeminne J, Seegers M, et al. ${ }^{18}$ FDG-positron emission tomography for the early prediction of response in advanced soft tissue sarcoma treated with imatinib mesylate (Glivec). Eur J Cancer. 2003;39:2012-2020.

39. Van den Abbeele AD, Badawi RD. Use of positron emission tomography in oncology and its potential role to assess response to imatinib mesylate therapy in gastrointestinal stromal tumors (GISTs). Eur J Cancer. 2002;38(suppl 5): S60-S65.

40. Antoch G, Kanja J, Bauer S, et al. Comparison of PET, CT, and dual-modality PET/CT imaging for monitoring of imatinib (STI571) therapy in patients with gastrointestinal stromal tumors. J Nucl Med. 2004;45:357-365.

41. Gayed I, Vu Y, Iyer R, et al. The role of ${ }^{18} \mathrm{~F}$-FDG PET in staging and early prediction of response to therapy of recurrent gastrointestinal stromal tumors. $J$ Nucl Med. 2004;45:17-21.

42. Jager PL, Gietma JA, van der Graaf WT. Imatinib mesylate for the treatment of gastrointestinal stromal tumours: best monitored with FDG PET. Nucl Med Commun. 2004;25:433-438.

43. McAuliffe JC, Hunt KK, Lazar AJ, et al. A randomized, phase II study of preoperative plus postoperative imatinib in GIST: evidence of rapid radiographic response and temporal induction of tumor cell apoptosis. Ann Surg Oncol. 2009;16:910-919.

44. Blay JY, Bonvalot S, Casali P, et al. Consensus meeting for the management of gastrointestinal stromal tumors: report of the GIST Consensus Conference of 2021 March 2004, under the auspices of ESMO. Ann Oncol. 2005;16:566-578.

45. Schuetze SM. Imaging and response in soft tissue sarcomas. Hematol Oncol Clin North Am. 2005;19:471-487. 
46. Schuetze SM. Utility of positron emission tomography in sarcomas. Curr Opin Oncol. 2006;18:369-373.

47. Hicks RJ. Functional imaging techniques for evaluation of sarcomas. Cancer Imaging. 2005;5:58-65.

48. Khamly KK, Hicks RJ, McArthur GA, Thomas DM. The promise of PET in clinical management and as a sensitive test for drug cytotoxicity in sarcomas. Expert Rev Mol Diagn. 2008;8:105-119.

49. Toner GC, Hicks RJ. PET for sarcomas other than gastrointestinal stromal tumors. Oncologist. 2008;13(suppl 2):22-26.

50. Schuetze SM, Rubin BP, Vernon C, et al. Use of positron emission tomography in localized extremity soft tissue sarcoma treated with neoadjuvant chemotherapy. Cancer. 2005;103:339-348.

51. Hawkins DS, Schuetze SM, Butrynski JE, et al. $\left[{ }^{18} \mathrm{~F}\right]$ fluorodeoxyglucose positron emission tomography predicts outcome for Ewing sarcoma family of tumors. J Clin Oncol. 2005;23:8828-8834.

52. Schulte M, Brecht-Krauss D, Werner M, et al. Evaluation of neoadjuvant therapy response of osteogenic sarcoma using FDG PET. J Nucl Med. 1999;40:16371643.

53. Sato J, Yanagawa T, Dobashi Y, Yamaji T, Takagishi K, Watanabe H. Prognostic significance of ${ }^{18} \mathrm{~F}$-FDG uptake in primary osteosarcoma after but not before chemotherapy: a possible association with autocrine motility factor/ phosphoglucose isomerase expression. Clin Exp Metastasis. 2008;25:427435.

54. Ye Z, Zhu J, Tian M, et al. Response of osteogenic sarcoma to neoadjuvant therapy: evaluated by ${ }^{18}$ F-FDG-PET. Ann Nucl Med. 2008;22:475-480.

55. Hawkins DS, Rajendran JG, Conrad EU III, Bruckner JD, Eary JF. Evaluation of chemotherapy response in pediatric bone sarcomas by [F-18]-fluorodeoxy-Dglucose positron emission tomography. Cancer. 2002;94:3277-3284.
56. Franzius $\mathrm{C}$, Schober O. Assessment of therapy response by FDG PET in pediatric patients. Q J Nucl Med. 2003;47:41-45.

57. McCarville MB, Christie R, Daw NC, Spunt SL, Kaste SC. PET/CT in the evaluation of childhood sarcomas. AJR. 2005;184:1293-1304.

58. Krohn KA, Link JM, Mason RP. Molecular imaging of hypoxia. J Nucl Med. 2008;49(suppl 2):129S-148S.

59. Rajendran JG, Wilson DC, Conrad EU, et al. $\left[{ }^{18} \mathrm{~F}\right]$ FMISO and $\left[{ }^{18} \mathrm{~F}\right]$ FDG PET imaging in soft tissue sarcomas: correlation of hypoxia, metabolism and VEGF expression. Eur J Nucl Med Mol Imaging. 2003;30:695-704.

60. Tatum JL, Kelloff GJ, Gillies RJ, et al. Hypoxia: importance in tumor biology, noninvasive measurement by imaging, and value of its measurement in the management of cancer therapy. Int J Radiat Biol. 2006;82:699-757.

61. Kelloff GJ, Krohn KA, Larson SM, et al. The progress and promise of molecular imaging probes in oncologic drug development. Clin Cancer Res. 2005;11:79677985 .

62. Cobben DC, Elsinga PH, Suurmeijer AJ, et al. Detection and grading of soft tissue sarcomas of the extremities with ${ }^{18} \mathrm{~F}$-3'-fluoro-3'-deoxy-L-thymidine. Clin Cancer Res. 2004;10:1685-1690.

63. Buck AK, Herrmann K, Buschenfelde CM, et al. Imaging bone and soft tissue tumors with the proliferation marker $\left[{ }^{18} \mathrm{~F}\right]$ fluorodeoxythymidine. Clin Cancer Res. 2008;14:2970-2977.

64. Been LB, Suurmeijer AJ, Elsinga PH, Jager PL, van Ginkel RJ, Hoekstra HJ. ${ }^{18} \mathrm{~F}$-fluorodeoxythymidine PET for evaluating the response to hyperthermic isolated limb perfusion for locally advanced soft-tissue sarcomas. J Nucl Med. 2007;48:367-372. 